\title{
Isolated Taylor Bubbles in Co-Current with Shear Thinning CMC Solutions in Microchannels- A Numerical Study
}

\author{
Ana I. Moreira ${ }^{1}$, Luís A. M. Rocha ${ }^{2}$, João Carneiro ${ }^{1}$, José D. P. Araújo ${ }^{1}$, João B. L. M. Campos ${ }^{1}$ \\ and João M. Miranda 1,*iC \\ 1 CEFT-Transport Phenomena Research Center, Chemical Engineering Department, Faculty of Engineering, \\ University of Porto, 4200-465 Porto, Portugal; aidm@fe.up.pt (A.I.M.); jcarneiro@fe.up.pt (J.C.); \\ daraujo@fe.up.pt (J.D.P.A.); jmc@fe.up.pt (J.B.L.M.C.) \\ 2 Department of Chemical Engineering and Biotechnology, University of Cambridge, \\ Cambridge CB3 0AS, UK; lam99@cam.ac.uk \\ * Correspondence: jmiranda@fe.up.pt
}

Received: 8 January 2020; Accepted: 13 February 2020; Published: 20 February 2020

\begin{abstract}
Slug flow is a multiphase flow pattern characterized by the occurrence of long gas bubbles (Taylor bubbles) separated by liquid slugs. This multiphase flow regime is present in many and diversified natural and industrial processes, at macro and microscales, such as in eruption of volcanic magmas, oil recovery from pre-salt regions, micro heat exchangers, and small-sized refrigerating systems. Previous studies in the literature have been mostly focused on tubular gas bubbles flowing in Newtonian liquids. In this work, results from several numerical simulations of tubular gas bubbles flowing in a shear thinning liquid in microchannels are reported. To simulate the shear thinning behavior, carboxymethylcellulose (CMC) solutions with different concentrations were considered. The results are compared with data from bubbles flowing in Newtonian liquids in identical geometric and dynamic conditions. The numerical work was carried out in computational fluid dynamics (CFD) package Ansys Fluent (release 16.2.0) employing the volume of fluid (VOF) methodology to track the volume fraction of each phase and the continuum surface force (CSF) model to insert the surface tension effects. The flow patterns, the viscosity distribution in the liquid, the liquid film thickness between the bubble and the wall, and the bubbles shape are analyzed for a wide range of shear rates. In general, the flow patterns are similar to those in Newtonian liquids, but in the film, where a high viscosity region is observed, the thickness is smaller. Bubble velocities are smaller for the non-Newtonian cases.
\end{abstract}

Keywords: shear thinning fluids; Taylor bubbles; microfluidics; carboxymethylcellulose solutions; volume of fluid method; computational fluid dynamics

\section{Introduction}

Gas-liquid slug flow, also known as Taylor bubble flow, is a multiphase flow pattern characterized by the presence of long bullet-shaped bubbles, occupying almost all of the cross-section of the tube, separated by stagnant liquid or by liquid flowing co-currently or counter-currently [1-5]. In the most common case, the Taylor bubble outruns the liquid phase leading to the development of a liquid film between the lateral surface of the bubble and the channel wall [1-3,6,7]. This flow pattern can occur in channels of different shapes (e.g., square, circular, or triangular cross section), dimensions (micro or macroscale) [1] and orientations (horizontal, vertical, and inclined channels) [3].

Gas-liquid slug flows have an important impact in many industrial processes, such as the oil industry [7], coating [7], micro heat exchangers [7], and small-sized refrigerating systems [8], where, 
most of the times, the bubbles enhance mass and heat transfer rates $[1,7,9,10]$. More specifically, due to the particular hydrodynamic features involved, the movement of a Taylor bubble can induce mixing in the surrounding liquid, making the flow more suitable for several physical and/or chemical processes $[4,8,11]$. On the other hand, when insufficiently controlled, the randomness and intermittence of the slug flow can cause severe over-pressurization issues [12] and, in corrosive environments, the corresponding wall shear stress fluctuations can lead to the erosion and cracking of metal surfaces [13]. Taylor bubbles are also important in several microscale biological and medical systems, as is the case of the development of gas embolisms [14] and control of biofilm formation [15,16]. Bubbles are also used as contrast agents [17] in medical diagnosis and as carriers for drug release and may acquire the typical Taylor bubble shape in the smallest capillary vessels. Several of the aforementioned scenarios may include gas-liquid slug flow with a non-Newtonian continuous phase $[18,19]$, thus prompting the need to characterize in detail the hydrodynamics involved. Due to the spatial and temporal variation of shear stresses in slug flow, and the corresponding direct effect on the viscosity fields, this characterization entails an extra degree of complexity. Nevertheless, this challenge has been already addressed numerically for macroscale systems [20], but a systematic study for microscale systems is still lacking.

In multiphase flows, the hydrodynamics is governed by the interaction of gravitational, interfacial, viscous and inertial forces. This interaction is highly dependent on the systems dimensions, i.e., the dominant forces at the macroscale may not be the dominant at the microscale. At the microscale, surface tension and viscosity have a great influence on the flow patterns, while gravity has little or no influence and can be neglected. The Eötvös number (Eo) is a characteristic dimensionless group that is commonly used to help delimit the referred dominance since it relates interfacial and gravitational forces:

$$
\mathrm{Eo}=\frac{\left(\rho_{L}-\rho_{g}\right) g D^{2}}{\sigma}
$$

where $\rho_{L}$ is the liquid density, $\rho_{g}$ the gas density, $g$ the gravitational acceleration, $D$ the diameter of the channel, and $\sigma$ the surface tension. According to Suo and Griffith [21], the gravitational forces are negligible for Eo $<(2 \pi)^{2}$, while Bretherton [22] claims that this occurs for Eo $<3.37$. This means that gas-liquid flows in microchannels and small diameter milliscale channels are not influenced by gravity and the flow is independent of the channel orientation. Thus, gas-liquid flows at the microscale are governed by surface tension, viscosity, and inertial forces, i.e., hydrodynamic features like the normalized bubble velocity depend on a small set of the dimensionless groups: the Capillary and Reynolds number of one of the phases. The Capillary number relates viscous and surface tension forces:

$$
\mathrm{Ca}_{\mathrm{i}}=\frac{\mu_{i} v_{i}}{\sigma}
$$

where $\mathrm{Ca}_{\mathrm{i}}$ is the capillary number of the phase $i, \mu_{i}$ the viscosity of the phase $i$ and $v_{i}$ the velocity of the phase $i$. When the capillary number is high the viscosity effects dominate over the surface tension effects. The Reynolds number quantifies the ratio between the inertial forces and the viscous forces:

$$
\operatorname{Re}_{\mathrm{i}}=\frac{\rho_{i} v_{i} D}{\mu_{i}}
$$

where $\operatorname{Re}_{i}$ is the Reynolds number of the phase $i$, and $\rho_{i}$ the density of the phase $i$. At the microscale, Reynolds number is usually low. It is noteworthy that gas-liquid flows are not influenced by the viscosity ratio between phases because the viscosity of the gas is negligible when compared with the viscosity of the liquid.

Since gas-liquid slug flow in microchannels also depends on the viscous forces, it is crucial to understand the differences on the behavior of Taylor bubbles and their surroundings when the 
liquid phase is Newtonian or non-Newtonian. Particular focus should be placed on the effect of shear thinning rheology.

Several authors have studied slug flow in non-Newtonian liquids in macroscale systems. Most of the works focused on the effect of shear thinning/thickening and viscoelasticity on bubble shape and velocity fields surrounding the bubble. More particularly, Sousa et al. [4] used particle image velocimetry (PIV) and shadowgraphy techniques to analyze the velocity fields around Taylor bubbles rising in carboxymethylcellulose (CMC) solutions. The authors observed, at low values of Reynolds number, a small closed liquid wake attached to the bubble bottom. The smaller size of the wake, when compared to the Newtonian counterpart, was attributed to the higher viscosity of the liquid in this region. Sousa et al. [23] also studied the interactions between Taylor bubbles flowing either in carboxymethylcellulose (CMC) solutions or in polyacrylamide (PAA) solutions. For low concentrations of $\mathrm{CMC}$, the results were similar to those obtained with Newtonian fluids in the same viscosity range. For concentrated solutions of $\mathrm{CMC}$, which imply higher viscosity levels and some elasticity, a negative wake was observed behind the bubble preventing bubbles coalescence. For PAA solutions, the role of elasticity was higher, leading to the formation of longer wakes. Araújo et al. [20] studied, numerically, the flow surrounding Taylor bubbles rising through inelastic non-Newtonian fluids. The authors analyzed the effect of shear thinning and shear thickening on the bubble shape and on the velocity field in each flow region around the bubble (around the nose, liquid film and wake). More recently, Majumdar and Das [24] studied, by analytical and numerical methods, the bubble shape and velocity and the velocity field surrounding Taylor bubbles rising in power law fluids.

The interest on microfluidics has led the research community to focus on the flow of Taylor bubbles in microchannels. The research has been focused on the effect of shear thinning/thickening and viscoelasticity on flow pattern maps, bubble shape, and velocity. Zhang et al. [25] studied the influence of liquid properties in gas-liquid flows using different concentrations of CMC- $0.0464 \%$ $(\mathrm{w} / \mathrm{w}), 0.1262 \%(\mathrm{w} / \mathrm{w})$, and $0.2446 \%(\mathrm{w} / \mathrm{w})$ and circular channels with different diameters $(302,496$, and $916 \mu \mathrm{m})$. The authors constructed flow pattern maps based on observations of bubbly, slug, slug-annular, annular, and churn flow regimes. The transition between the regimes is influenced by the liquid viscosity, the surface tension, and the channel size. Based on this dependence, the authors proposed a new model to predict regime transitions. The authors also observed that, in slug flow, the liquid film thickness increases with increasing CMC concentration. Yang et al. [26] conducted a study of gas-liquid flow in non-circular microchannels with square and triangular cross sections and hydraulic diameters of 2.886, 2.5, and $0.866 \mathrm{~mm}$. The gas phase was nitrogen and three different non-Newtonian fluids were tested: aqueous solutions of CMC, polyacrylamide (PAA), and xanthan gum (XG). The authors were able to obtain the flow pattern maps for the different systems and concluded that the transitions between the flow regimes is influenced by the channel geometry and the rheology of the non-Newtonian solutions. Mansour et al. [27] used polyacrylamide solutions to investigate gas/non-Newtonian liquid (PAA solutions) flows in a rectangular microchannel and compared the results with those from gas/Newtonian liquid flows. The authors found that the shape of the bubbles change due to the higher viscosity of the non-Newtonian liquid, inducing a thicker liquid film around the bubble. The authors also noted that higher concentrations of polyacrylamide lead to higher bubble velocities. Fu et al. [28] studied the formation of bubbles in a microchannel using also PAA solutions. The authors studied gas-liquid continuous slug flow, i.e., both phases are continuously injected and flow co-currently in the microchannel forming a segmented patterned of alternated Taylor bubbles and liquid slugs also known as bubble train flow. For this flow pattern, the size of the bubbles increased with the increase of the gas flow rate but diminished with the increase of the liquid flow rate. Furthermore, these tendencies were almost independent of the rheological properties of the solutions.

Concerning the prediction of the slug flow's main hydrodynamic features in microscale systems, several authors established correlations for the bubble velocity and liquid film thickness for systems with Newtonian fluids (Table 1). However, no equivalent correlations were found in the literature for 
systems with non-Newtonian fluids. The only exception is the equation to estimate the bubble velocity based on the stagnant liquid film hypothesis $[21,29,30]$ :

$$
V_{B}=\frac{1}{\left(1-\frac{\delta}{R}\right)^{2}} \bar{V}_{L}
$$

where $V_{B}$ is the bubble velocity, $\bar{V}_{L}$ the mean liquid velocity, $\delta$ the liquid film thickness, and $R$ the radius of the channel. The subscripts $L$ and $G$ are used, throughout the paper, for the liquid and gas phases, respectively. Equation (4) is valid for both Newtonian and non-Newtonian fluids.

Table 1. Film thickness and velocity correlations for bubbles flowing in Newtonian fluids.

\begin{tabular}{|c|c|c|c|}
\hline Parameter & Correlation & & Reference \\
\hline \multirow{4}{*}{$\begin{array}{l}\text { Dimensionless } \\
\text { film thickness }\end{array}$} & $1.34 \mathrm{Ca}^{\frac{2}{3}}$ & (5) & Bretherton [22] \\
\hline & \multirow{3}{*}{$0.5 \mathrm{Ca}^{\frac{1}{2}}$} & \multirow{3}{*}{ (6) } & Fairbrother and Stubbs [31] \\
\hline & & & Taylor [32] \\
\hline & & & Chen [33] \\
\hline \multirow{3}{*}{$\frac{\delta}{D}$} & $0.36\left[1-\exp \left(-3.08\left(\mathrm{Ca}^{0.54}\right)\right)\right]$ & (7) & Irandoust and Andersson [34] \\
\hline & $\frac{\left(1.34 \mathrm{Ca}^{\frac{2}{3}}\right)}{1+2.5\left(1.34 \mathrm{Ca}^{\frac{2}{3}}\right)}$ & (8) & Aussillous and Quéré [35] \\
\hline & $\frac{0.67 \mathrm{Ca}^{\frac{2}{3}}}{1+3.13 \mathrm{Ca}^{\frac{2}{3}}+0.504 \mathrm{Ca}^{0.672} \mathrm{Re}^{0.589}-0.352 \mathrm{We}^{0.629}}$ & $(9)$ & Han and Shikazono [36] \\
\hline Bubble velocity & $\frac{U_{T P}}{1-0.61 C a^{0.33}}$ & $(10)$ & Liu et al. [37] \\
\hline$V_{B}$ & $\begin{array}{c}\frac{V_{B}}{V_{T P}}=1+d\left[1-\exp \left(-\mathrm{F}\left(\mathrm{Ca}_{\mathrm{B}}\right)\right)\right] \\
F_{1}\left(\mathrm{Ca}_{\mathrm{B}}\right)=\exp \left[b+c \ln \left(\mathrm{Ca}_{\mathrm{B}}\right)\right] \\
F_{2}\left(\mathrm{Ca}_{\mathrm{B}}\right)=\exp \left[b+c \ln \left(\mathrm{Ca}_{\mathrm{B}}\right)+g \ln \left(\mathrm{Ca}_{\mathrm{B}}\right)^{2}\right]\end{array}$ & $(11)$ & Abiev and Lavretsov [38] \\
\hline
\end{tabular}

In summary, to our knowledge, there is a clear lack of reported information about non-Newtonian slug flow in microchannels. Considering the relevance of such systems, this is an important research gap that need to be filled and this work intends to be a step towards that goal. So, the focus of this work is to analyze microscale slug flow in shear thinning CMC solutions with the help of CFD tools. CMC solutions were selected as a case study mainly because of their wide application in two phase studies involving non-Newtonian. The use of CFD methodologies allowed to describe the complexity of the chosen systems with a high level of spatial and temporal detail. The differences between Newtonian slug flow and non-Newtonian shear thinning slug flow will be highlighted.

\section{Materials and Methods}

A set of simulations was performed to predict the flow of single Taylor bubbles moving through co-current non-Newtonian liquids inside circular microchannels. The non-Newtonian liquids addressed are inelastic (Deborah number below 0.001) and have shear thinning behavior, and this numerical study was performed for Capillary numbers between 0.02 and 0.17 . The commercial CFD package ANSYS Fluent ${ }^{\circledR}$ (Release 16.2.0) was the software chosen for this study following the approach of Rocha et al. [39]. The volume of fluid (VOF) methodology [40] included on this package was used as the gas-liquid interface tracking technique coupled with the geometric reconstruction scheme [41] that is based on a piecewise-linear approach to define the interface between phases.

\subsection{Domain and Numerical Method}

Due to the small dimensions involved, the flow in microscales is usually laminar (low Reynolds number), and so, for a circular microchannel, it is feasible to assume that the flow is axisymmetric In order to reduce the computational effort and time, the axisymmetric physical domain is mapped 
into a 2D rectangular mathematical domain. The numerical domain defined for a microchannel with a diameter of $100 \mu \mathrm{m}$ is represented in Figure 1; a rectangle with a length of $700 \mu \mathrm{m}$ and a width of $50 \mu \mathrm{m}$. Additionally, a domain 10 times longer was used to simulate the flow in the millichannels with a diameter of $1 \mathrm{~mm}$.

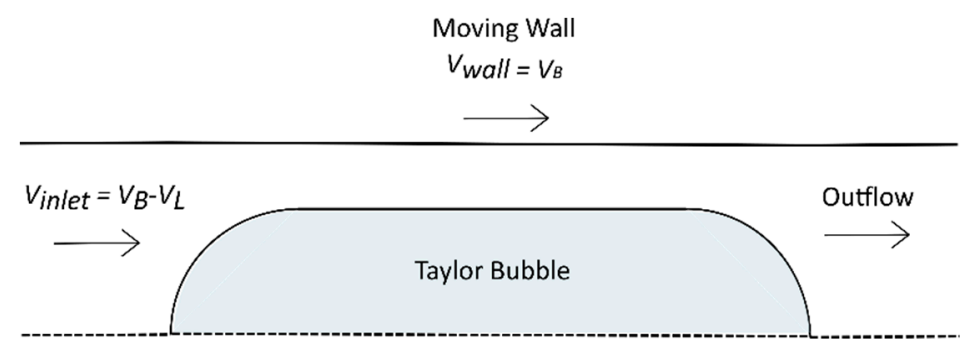

Figure 1. Domain of the simulations in a microchannel with a diameter of $100 \mu \mathrm{m}$.

In all the simulations, the ratio between the bubble volume and the area of the cross-section of the tube is constant in order to enable the comparison between bubble shape in different conditions. The liquid flows co-currently in the laminar regime, and so, at the inlet of the domain, a parabolic profile was imposed with the help of a user-defined function. At the outlet of the domain, the pressure was set to zero. A moving reference frame (MRF) was applied to reduce the length required for the domains: for each system, a velocity value was imposed to the channel wall (moving wall condition), which was equal to an estimated bubble velocity. This value was adjusted along the simulation until the bubble remained stationary in the domain, i.e., the position of the bubble nose tip stabilized (see Rocha et al. [39]).

\subsection{Governing Equations}

The continuity and momentum equations were solved to obtain the flow fields in both phases:

$$
\begin{gathered}
\frac{\partial \rho}{\partial t}+\nabla \cdot(\rho \vec{v})=0 \\
\frac{\partial(\rho \vec{v})}{\partial t}+\nabla \cdot(\rho \vec{v} \vec{v})=-\nabla p+\nabla \cdot\left[\mu\left(\nabla \vec{v}+\nabla^{T}\right)\right]+\vec{F}
\end{gathered}
$$

where $\vec{v}$ is the velocity vector, $t$ the time, $\rho$ the density, $\mu$ the viscosity, and $p$ the pressure.

The volume of fluid (VOF) method is based on the transport equation of the gas phase fraction, $\alpha_{G}$ :

$$
\frac{\partial \alpha_{G}}{\partial t}+\vec{v} \cdot \nabla \alpha_{G}=0
$$

The sum of the phase fractions is equal to 1 in any position of the domain:

$$
\alpha_{G}+\alpha_{L}=1
$$

In the momentum Equation (17), $\vec{F}$ represents the surface tension force and it was computed based on the continuum surface force (CSF) model [42]:

$$
F=\sigma \frac{\rho k_{G} \nabla \alpha_{G}}{\frac{1}{2}\left(\rho_{G}+\rho_{L}\right)}
$$

where $\sigma$ is the surface tension and $k_{G}$ the bubble curvature, computed from the divergence of the unit vector normal to the interface:

$$
k_{G}=\nabla \cdot \hat{\vec{n}}
$$


where $\vec{n}$ is calculated by

$$
\vec{n}=\nabla \alpha_{G}
$$

The density and viscosity are calculated through

$$
\begin{aligned}
& \rho=\alpha_{G} \rho_{G}+\left(1-\alpha_{G}\right) \rho_{L} \\
& \mu=\alpha_{G} \mu_{G}+\left(1-\alpha_{G}\right) \mu_{L}
\end{aligned}
$$

The continuity and momentum equations were solved through the PISO ("Pressure-implicit with splitting of operators") algorithm. To discretize the equation terms, the pressure interpolations were done through the pressure staggering option (PRESTO!), the convective terms were approximated by the "QUICK" scheme and the discretized gradients of the scalars were computed with the Green-Gauss method. A variable time step controlled by the imposition of a global Courant number of 0.25 was applied in the solution of the unsteady-state model previously defined. For each time step, a maximum number of iterations of 1000 was fixed, and the convergence criterion was supported on the scaled absolute values of the velocities and continuity residuals, which were all monitored and set to a maximum of $10^{-6}$.

\subsection{Fluids}

In order to study the behavior of Taylor bubbles moving in inelastic shear thinning liquids, $\mathrm{CMC}$ solutions of 0.10 and $0.50 \mathrm{wt}$. \% were selected for the continuous phase. The rheology of the CMC solutions was characterized through the Carreau-Yasuda model:

$$
\mu=\mu_{\infty}+\left(\mu_{0}-\mu_{\infty}\right)\left(1+(\lambda \cdot \dot{\gamma})^{a_{1}}\right)^{\frac{a_{2}-1}{a_{1}}}
$$

where $\mu$ is the fluid viscosity, $\dot{\gamma}$ the shear stress, $\mu_{0}$ the viscosity limit for shear stresses approaching zero, $\mu_{\infty}$ the fluid viscosity for an infinite shear stress, $\lambda$ a time constant, and $a_{1}$ and $a_{2}$ dimensionless parameters. This model was implemented in ANSYS Fluent ${ }^{\circledR}$ through a user-defined function. The values of the parameters in the Carreau-Yasuda model for the CMC solutions are reported in the work of Sousa et al. [4] and are listed in Table 2. The Deborah number for both solutions is below 0.001 [4], which means that they are practically inelastic.

Table 2. Carreau-Yasuda parameters values. Table adapted from Sousa et al. [4].

\begin{tabular}{ccccccc}
\hline \% CMC & $\mu_{0}$ (Pa.s) & $\mu_{\infty}$ (Pa.s) & $\lambda(\mathbf{s})$ & $a_{1}$ & $a_{2}$ & $\dot{\gamma}\left(\mathbf{s}^{-\mathbf{1}}\right)$ \\
\hline 0.10 & 0.009 & 0.001 & 0.021 & 0.850 & 0.871 & $1-4000$ \\
\hline 0.50 & 0.220 & 0.001 & 0.063 & 0.565 & 0.509 & $0.25-4000$ \\
\hline
\end{tabular}

\subsection{Non-Dimensional Parameters}

Capillary and Reynolds numbers for non-Newtonian fluids were calculated based on the characteristic viscosity, i.e., the viscosity of the fluid for the characteristic flow rate. The characteristic flow shear rate, $\dot{\gamma}_{f}$, was determined by

$$
\dot{\gamma}_{f}=\frac{\bar{V}_{L}}{D}
$$

\section{Results}

\subsection{Study Design}

The numerical study of Taylor bubbles moving in a shear-thinning CMC solution inside a microchannel is limited by constrains imposed by the fluid properties, the size of the channel, and the 
numerical method used. These constrains are illustrated in Figure 2. For concentrations above 0.5\%, $\mathrm{CMC}$ solutions become viscoelastic and so shear thinning effects cannot be singled out. On the other end, solutions of $0.1 \%$ CMC have a behavior very close to Newtonian. For low shear rates, the Capillary number can be too low, thus the simulations can be affected by numerical parasitic currents: as a reference, the lower limit is $\dot{\gamma}=100 \mathrm{~s}^{-1}$ for a $100 \mu \mathrm{m}$ tube and $\dot{\gamma}=10 \mathrm{~s}^{-1}$ for a $1 \mathrm{~mm}$ tube. For shear rates higher than $\mathrm{s}^{-1}$, a $0.5 \% \mathrm{CMC}$ solution has a constant viscosity and the shear thinning effects are not observable.

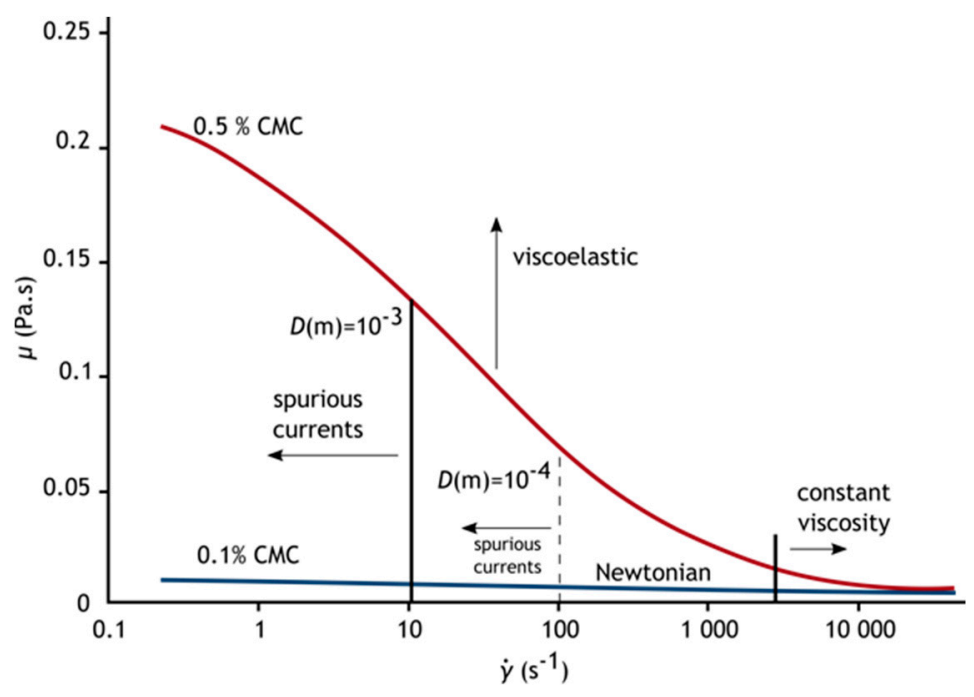

Figure 2. Viscosity of $\mathrm{CMC}$ solutions as a function of the shear rate, showing the main constrains to the study. Viscosity curves were calculated using Equation (20).

According to this analysis, different simulations were performed for $0.5 \% \mathrm{CMC}$ solutions above $10 \mathrm{~s}^{-1}$ characteristic shear rates. For this solution, the viscosity changes deterministically with the bubble velocity and, therefore, Capillary and Reynolds numbers are not independent parameters.

\subsection{Flow Fields}

The flow fields (velocity vectors and streamlines) obtained for different flow conditions in a tube with a diameter of $100 \mu \mathrm{m}$ are represented in Figure 3. These representations are based on a reference frame attached to the bubble (MRF). The simulations were carried out for liquid phase Capillary numbers ranging from 0.04 to 0.18 (corresponding to Reynolds numbers ranging from 0.37 to 17 ). For $\mathrm{Ca}>0.14$, the results concern high characteristic shear rates, in the region of constant viscosity (Figure 2). The typical flow behavior comprises semi-infinite recirculation zones downstream and upstream to the bubble, a thin liquid film between the bubble and the wall, a large vortex in the inside central region of the bubble, and several smaller ones on the inside ends of the bubble. As it can be seen, when the Capillary number increases, the bubble becomes elongated, the thickness of the film increases, and the bubble becomes front/back asymmetric. From a qualitative perspective, these results are similar to those obtained for similar systems with Newtonian fluids. For the same Capillary numbers, it can be observed that that the liquid film is thinner for the $0.5 \% \mathrm{CMC}$ solution, which has a shear thinning behavior more pronounced. 

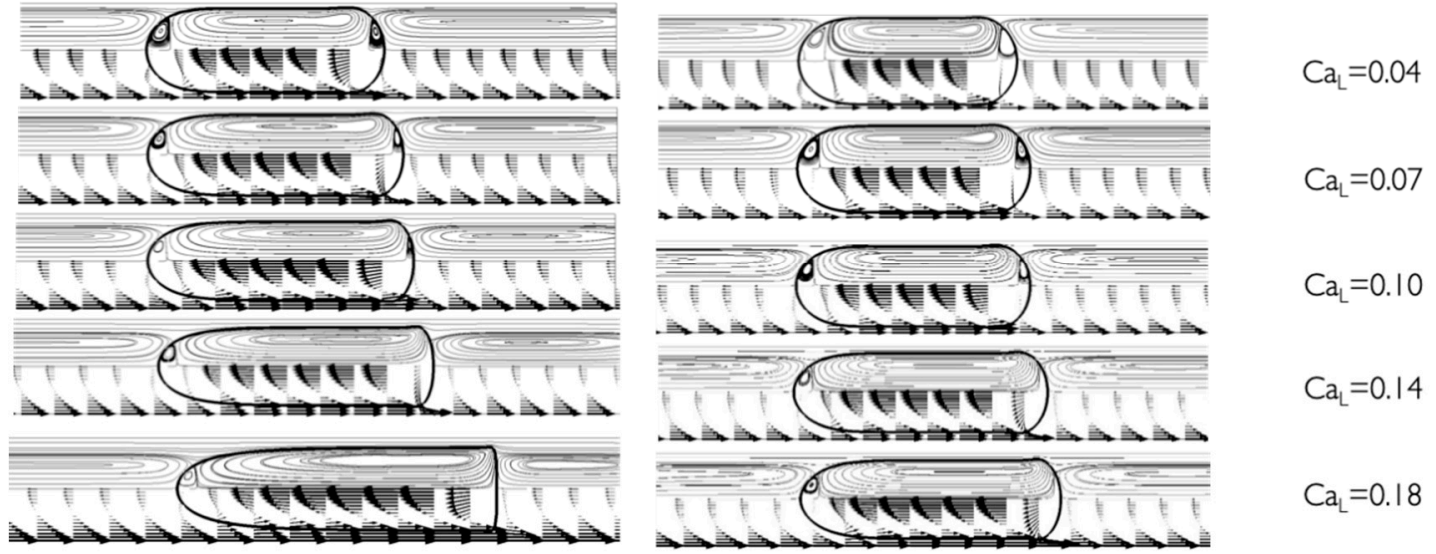

Figure 3. Numerical results of the velocity vectors and streamlines for systems with bubbles flowing through a $0.1 \% \mathrm{CMC}$ solution (left) and a $0.5 \% \mathrm{CMC}$ solution (right). The domain under consideration has a $D=100 \mu \mathrm{m}$.

\subsection{Viscosity Fields}

To better understand how the local viscosity changes in the bubble surroundings, as a consequence of the shear thinning effect, the viscosity fields obtained for several simulations are represented along the characteristic viscosity curve in Figure 4. Two features of the viscosity field can be observed: (1) the decrease of viscosity with the increase of the characteristic shear rate; and (2) the spatial variation of viscosity due to the spatial variation of the shear rate. The decrease of viscosity with the increase of the characteristic shear rate can be checked almost everywhere in the domain and is a consequence of the shear thinning behavior. Regarding the spatial variation of viscosity, it is important to notice the presence of some high viscosity spots in the axis of the channel and in the film region. The appearance of high viscosities in the film is due to liquid almost stagnated, and in axis of the channel it is due to a low velocity gradient.

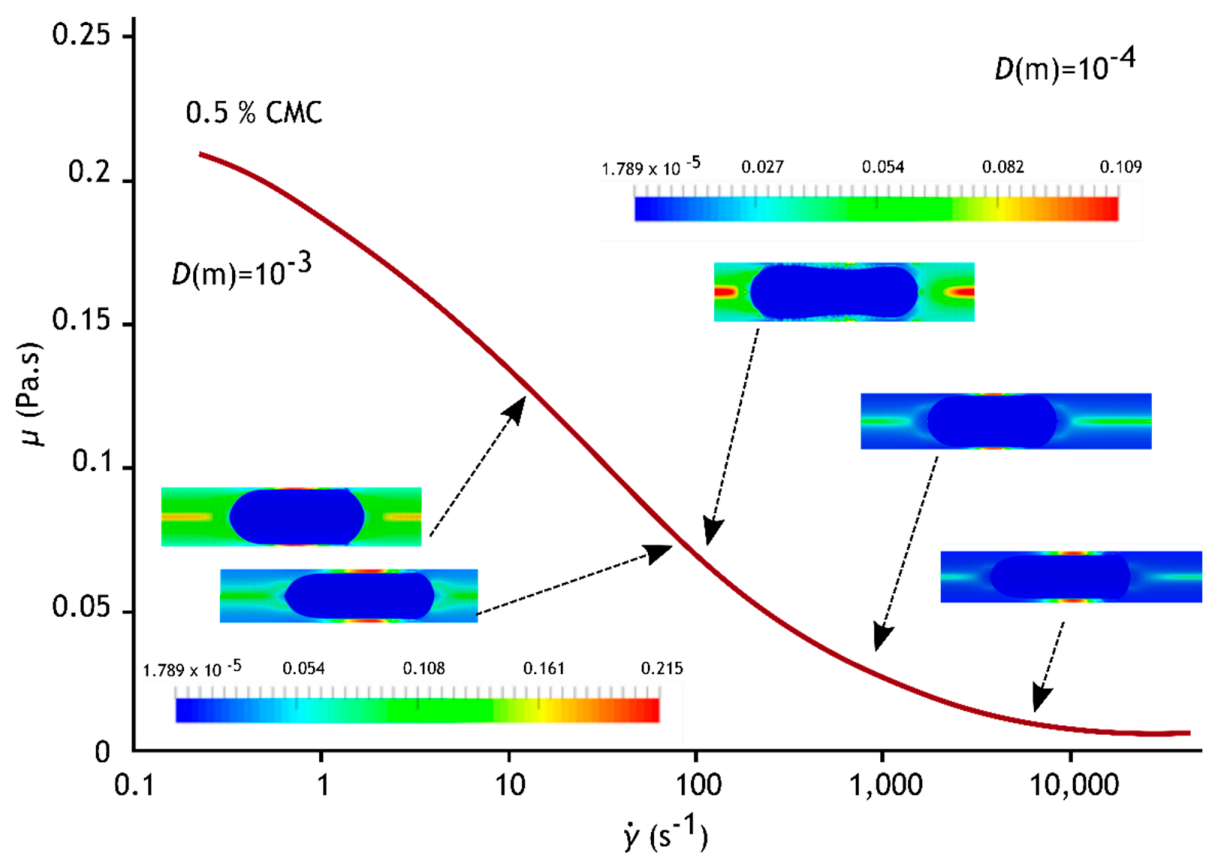

Figure 4. Viscosity fields along the characteristic viscosity curve of a $0.5 \%$ CMC solution obtained for microchannels with a $100 \mu \mathrm{m}$ and $1000 \mu \mathrm{m}$ diameter. The viscosity curve was calculated using Equation (20). 


\subsection{Bubble Velocity}

Taylor bubble velocity is one of the main hydrodynamic features of slug flow in microfluidics since it is directly related to the residence time of the gas phase in the device. In all the systems addressed, the bubble moves faster than the liquid phase. Figures 5 and 6 show the bubble velocity normalized by the average liquid velocity as a function of the liquid and bubble Capillary numbers, respectively. For the same Capillary number, the velocities obtained for $0.5 \%$ and $0.1 \%$ CMC solutions are always lower than those obtained for the corresponding flow of a Newtonian liquid. According to Figure 5, Liu et al.'s [37] correlation underpredicts the velocity of the bubbles in Newtonian liquids and overpredicts it in the $0.5 \% \mathrm{CMC}$ solution. The Abiev and Lavretsov [38] correlation overpredicts both (Figure 6), being more accurate for the Newtonian system.

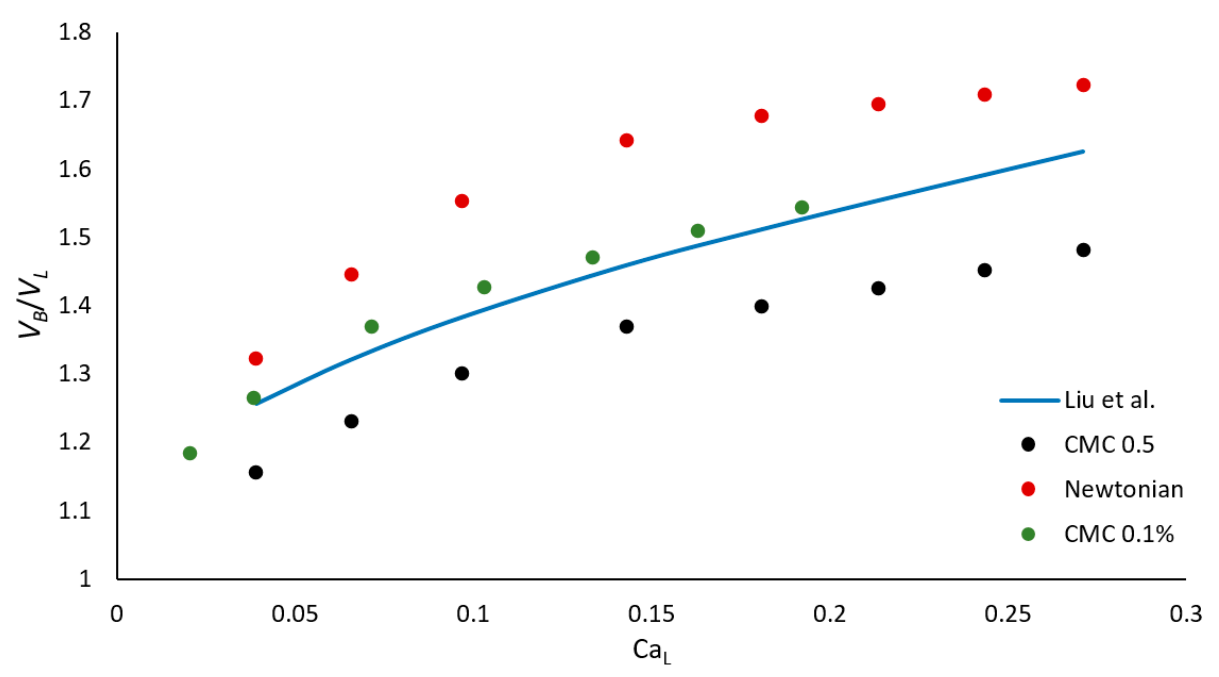

Figure 5. Bubble velocity normalized by the liquid velocity as a function of the liquid capillary number. The numerical results are for bubbles flowing in CMC solutions $(0.1 \%$ and $0.5 \%)$ and in a Newtonian liquid with a viscosity corresponding to the characteristic shear rate. The theoretical predictions were based on the Liu et al. [37] correlation. The presented numerical data concerns the systems with a channel of $D=100 \mu \mathrm{m}$.

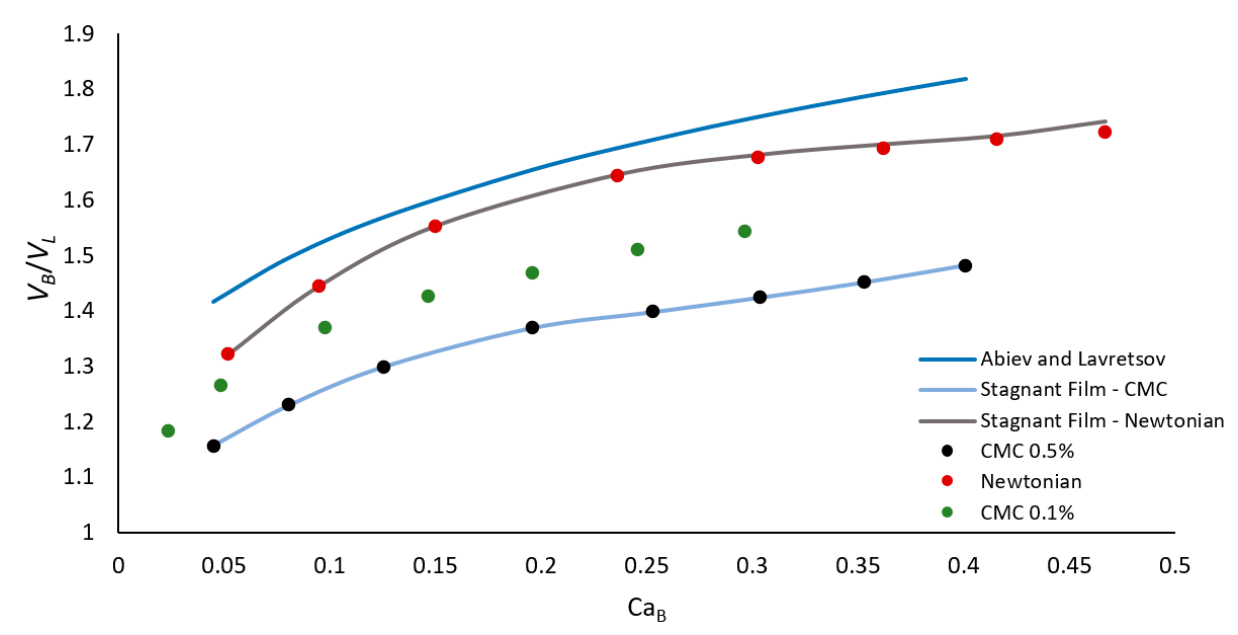

Figure 6. Bubble velocity normalized by the liquid velocity as a function of the bubble capillary number. The numerical results are for bubbles flowing in CMC solutions $(0.1 \%$ and $0.5 \%)$ and in a Newtonian liquid with a viscosity corresponding to the characteristic shear rate. The theoretical predictions are based on the stagnant film model and on the Abiev and Lavretsov [38] correlation. The numerical data concerns the systems with a channel of $D=100 \mu \mathrm{m}$. 
The main reason for the lower normalized velocity of bubbles in shear thinning fluids is the velocity profile of the liquid flow downstream to the bubble. In Newtonian fluids, the velocity profile is parabolic, and the maximum velocity is two times the average liquid velocity. In shear thinning fluids, the ratio between the maximum velocity and the average velocity is less than two. Due to volumetric flow rate conservation, the bubble must have a velocity similar to the liquid flowing downstream to the bubble, which is lower for shear thinning fluids.

The model based on the stagnant film takes the film thickness obtained numerically to predict the bubble velocity. As shown in Figure 6, this model is highly accurate for the case of the Newtonian system and for the $0.5 \%$ CMC solution. The stagnant film model is accurate because the velocity in the film is really very low, which is confirmed by the numerical results. This should be expected in the film around Taylor bubbles because gases have a viscosity at least two orders of magnitude lower than liquids. To assure shear stress continuity in the interface between the Taylor bubble and the surrounding liquid film, the film needs to be almost stagnant. In these conditions, volumetric flow rate conservation along the tube implies that the bubble must be faster than the average liquid velocity.

\subsection{Film Thickness}

Another important hydrodynamic feature in the flow of Taylor microbubbles is the film thickness. This feature is directly linked to the bubble velocity and has important implications in the behavior of the vortexes up and downstream to the bubble and, consequently, in mass and heat transport enhancement due to the bubble movement. According to Figure 7, the equation developed by Han and Shikazono [36] predicts well the film thickness for bubbles flowing in Newtonian fluids. This figure also shows that the normalized film is thinner for bubbles flowing in shear thinning liquid when compared to their Newtonian counterparts and that the normalized thickness of the film decreases as the $\mathrm{CMC}$ concentration increases.

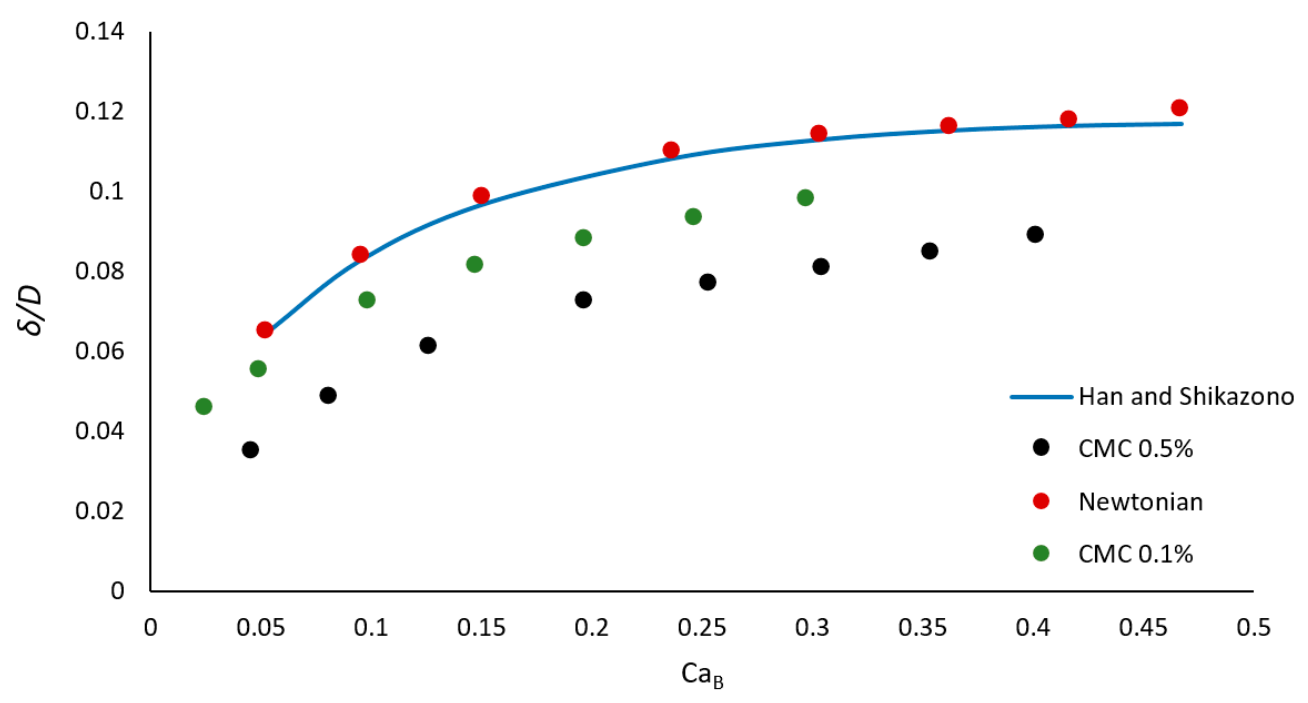

Figure 7. Normalized film thickness as a function of the bubble capillary number. Numerical results are for bubbles flowing in CMC solutions $(0.1 \%$ and $0.5 \%)$ and Newtonian liquid with a viscosity corresponding to the characteristic shear rate. The theoretical predictions are based on the Han and Shikazono [36] correlation. The numerical data concerns the systems with a channel of $D=100 \mu \mathrm{m}$.

\section{Conclusions}

The flow of single Taylor bubbles in co-current with shear thinning CMC solutions inside microchannels was numerically studied with the help of the VOF method. The range of conditions addressed was restricted by the limitations of the VOF method for systems with low Capillary number, and by the appearance of viscoelastic effects for CMC concentrations above $0.5 \%$. The study was then 
conducted for a $0.5 \% \mathrm{CMC}$ aqueous solution, for which rheological data is available and the shear thinning behavior can be observed with negligible viscoelastic effects.

The hydrodynamics was studied in systems that produced shear rates in two regions of the viscosity curve, i.e., the shear thinning region and the high shear constant viscosity region. In the shear thinning region, it is possible to observe the corresponding non-Newtonian effects on the viscosity distribution, for example, a high viscosity region in the liquid film.

In order to compare numerical results between systems with a Newtonian and non-Newtonian continuous phase, it was necessary to define the Capillary number for the non-Newtonian liquid flow. In this work, the Capillary number was defined by taking the viscosity for the characteristic shear rate of the flow, and Newtonian liquids with the same viscosity were assumed as the reference to perform the referred comparisons. The results show that for the same Capillary number, Taylor bubbles flowing in shear thinning liquids have a smaller film thickness and smaller velocity. Most of the correlations available in the literature were developed for Newtonian continuous phases. The exception is the correlation based on the stagnant film model, which is accurate for non-Newtonian fluids but requires the film thickness as input.

To further understand the flow of Taylor bubbles in microchannels, future works must address shear thinning fluids alternative to CMC, shear thickening fluids, and flow conditions dominated by viscoelasticity.

Author Contributions: Conceptualization, J.B.L.M.C. and J.M.M.; methodology, J.B.L.M.C; software, A.I.M., L.A.M.R. and J.C.; investigation, A.I.M.; resources, J.B.L.M.C. and J.M.M.; data curation, A.I.M.; writing-original draft preparation, A.I.M.; writing-review and editing, J.B.L.M.C., J.D.P.A. and J.M.M.; visualization, A.I.M.; supervision, J.B.L.M.C. and J.M.M.; project administration, J.B.L.M.C. and J.M.M.; funding acquisition, J.B.L.M.C. and J.M.M. All authors have read and agreed to the published version of the manuscript.

Funding: This work was funded by FEDER funds through COMPETE2020-Operational Programme for Competitiveness Factors (POCI) and National Funds (PIDDAC) through FCT (Fundação para a Ciência e a Tecnologia) under projects POCI-01-0145-FEDER-031758, POCI-01-0145-FEDER-016861-PTDC/QEQ-FTT/4287/2014 and PD/BD/114313/2016 and Transport Phenomena Research Center (CEFT) base funding (UIDB/00532/2020).

Conflicts of Interest: The authors declare no conflict of interest.

\section{References}

1. Thulasidas, T.C.; Abraham, M.A.; Cerro, R.L. Flow patterns in liquid slugs during bubble-train flow inside capillaries. Chem. Eng. Sci. 1997, 52, 2947-2962. [CrossRef]

2. Thulasidas, T.C.; Abraham, M.A.; Cerro, R.L. Dispersion during bubble-train flow in capillaries. Chem. Eng. Sci. 1999, 54, 61-76. [CrossRef]

3. Taha, T.; Cui, Z.F. Hydrodynamics of slug flow inside capillaries. Chem. Eng. Sci. 2004, 59, 1181-1190. [CrossRef]

4. Sousa, R.G.; Riethmuller, M.L.; Pinto, A.M.F.R.; Campos, J.B.L.M. Flow around individual Taylor bubbles rising in stagnant CMC solutions: PIV measurements. Chem. Eng. Sci. 2005, 60, 1859-1873. [CrossRef]

5. Morgado, A.O.; Miranda, J.M.; Araújo, J.D.P.; Campos, J.B.L.M. Review on vertical gas-liquid slug flow. Int. J. Multiph. Flow 2016, 85, 348-368. [CrossRef]

6. Nigmatulin, T.R.; Bonetto, F.J. Shape of Taylor bubbles in vertical tubes. Int. Commun. Heat Mass Transf. 1997, 24, 1177-1185. [CrossRef]

7. Han, Y.; Shikazono, N. The effect of bubble acceleration on the liquid film thickness in micro tubes. Int. J. Heat Fluid Flow 2010, 31, 630-639. [CrossRef]

8. Triplett, K.A.; Ghiaasiaan, S.M.; Abdel-Khalik, S.I.; Sadowski, D.L. Gas-liquid two-phase flow in microchannels Part I: Two-phase flow patterns. Int. J. Multiph. Flow 1999, 25, 377-394. [CrossRef]

9. Thulasidas, T.C.; Abraham, M.A.; Cerro, R.L. Bubble-train flow in capillaries of circular and square cross section. Chem. Eng. Sci. 1995, 50, 183-199. [CrossRef]

10. Han, Y.; Shikazono, N. Measurement of the liquid film thickness in micro tube slug flow. Int. J. Heat Fluid Flow 2009, 30, 842-853. [CrossRef]

11. Bugg, J.D.; Mack, K.; Rezkallah, K.S. A numerical model of Taylor bubbles rising through stagnant liquids in vertical tubes. Int. J. Multiph. Flow 1998, 24, 271-281. [CrossRef] 
12. Malekzadeh, R.; Henkes, R.; Mudde, R. Severe slugging in a long pipeline-riser system: Experiments and predictions. Int. J. Multiph. Flow 2012, 46, 9-21. [CrossRef]

13. Nešić, S. Key issues related to modelling of internal corrosion of oil and gas pipelines-A review. Corros. Sci. 2007, 49, 4308-4338. [CrossRef]

14. Bento, D.; Sousa, L.; Yaginuma, T.; Garcia, V.; Lima, R.; Miranda, J.M. Microbubble moving in blood flow in microchannels: Effect on the cell-free layer and cell local concentration. Biomed. Microdevices 2017, $19,6$. [CrossRef] [PubMed]

15. Silva, M.C.; Araújo, J.D.; Campos, J.B. CFD studies coupling hydrodynamics and solid-liquid mass transfer in slug flow for matter removal from tube walls. AIChE J. 2017, 63, 2420-2439. [CrossRef]

16. Silva, M.; Campos, J.; Araújo, J. Mass transfer from a soluble wall into gas-liquid slug flow in a capillary tube. Int. J. Heat Mass Transf. 2019, 132, 745-761. [CrossRef]

17. Stride, E.; Saffari, N. Microbubble ultrasound contrast agents: A review. Proc. Inst. Mech. Eng. H 2003, 217, 429-447. [CrossRef]

18. Laporte, M.; Montillet, A.; Belkadi, A.; Della Valle, D.; Loisel, C.; Riaublanc, A.; Hauser, J. Investigation of gas/shear-thinning liquids flow at high throughput in microchannels with the aim of producing biosourced foam. Chem. Eng. Process. Process Intensif. 2019, 148, 107787. [CrossRef]

19. Bento, D.; Lopes, S.; Maia, I.; Pereira, A.; Fernandes, C.; Miranda, J.; Lima, R. Blood Flow of Bubbles Moving in Microchannels with Bifurcations. In Proceedings of the ECCOMAS Thematic Conference on Computational Vision and Medical Image Processing, Porto, Portugal, 16-18 October 2019; pp. 571-577.

20. Araújo, J.; Miranda, J.; Campos, J. Taylor bubbles rising through flowing non-Newtonian inelastic fluids. J. Non-Newton. Fluid Mech. 2017, 245, 49-66. [CrossRef]

21. Suo, M.; Griffith, P. Two-Phase Flow in Capillary Tubes. J. Basic Eng. 1964, 86, 576-582. [CrossRef]

22. Bretherton, F.P. The motion of long bubbles in tubes. J. Fluid Mech. 1961, 10, 166-188. [CrossRef]

23. Sousa, R.G.; Pinto, A.M.F.R.; Campos, J.B.L.M. Interaction between Taylor bubbles rising in stagnant non-Newtonian fluids. Int. J. Multiph. Flow 2007, 33, 970-986. [CrossRef]

24. Majumdar, A.; Das, P. Rise of Taylor bubbles through power law fluids-Analytical modelling and numerical simulation. Chem. Eng. Sci. 2019, 205, 83-93. [CrossRef]

25. Zhang, T.; Cao, B.; Fan, Y.; Gonthier, Y.; Luo, L.; Wang, S. Gas-liquid flow in circular microchannel. Part I: Influence of liquid physical properties and channel diameter on flow patterns. Chem. Eng. Sci. 2011, 66, 5791-5803. [CrossRef]

26. Yang, Z.C.; Bi, Q.C.; Liu, B.; Huang, K.X. Nitrogen/non-Newtonian fluid two-phase upward flow in non-circular microchannels. Int. J. Multiph. Flow 2010, 36, 60-70. [CrossRef]

27. Mansour, M.H.; Kawahara, A.; Sadatomi, M. Experimental investigation of gas-non-Newtonian liquid two-phase flows from T-junction mixer in rectangular microchannel. Int. J. Multiph. Flow 2015, 72, 263-274. [CrossRef]

28. Fu, T.; Ma, Y.; Funfschilling, D.; Li, H.Z. Gas-liquid flow stability and bubble formation in non-Newtonian fluids in microfluidic flow-focusing devices. Microfluid. Nanofluidics 2011, 10, 1135-1140. [CrossRef]

29. Abadie, T.; Aubin, J.; Legendre, D.; Xuereb, C. Hydrodynamics of gas-liquid Taylor flow in rectangular microchannels. Microfluid. Nanofluidics 2012, 12, 355-369. [CrossRef]

30. Chaoqun, Y.; Yuchao, Z.; Chunbo, Y.; Minhui, D.; Zhengya, D.; Guangwen, C. Characteristics of slug flow with inertial effects in a rectangular microchannel. Chem. Eng. Sci. 2013, 95, 246-256. [CrossRef]

31. Fairbrother, F.; Stubbs, A.E. 119. Studies in electro-endosmosis. Part VI. The "bubble-tube" method of measurement. J. Chem. Soc. 1935, 527-529. [CrossRef]

32. Taylor, G.I. Deposition of a viscous fluid on the wall of a tube. J. Fluid Mech. 2006, 10, 161-165. [CrossRef]

33. Chen, J.-D. Measuring the film thickness surrounding a bubble inside a capillary. J. Colloid Interface Sci. 1986, 109, 341-349. [CrossRef]

34. Irandoust, S.; Andersson, B. Liquid film in Taylor flow through a capillary. Ind. Eng. Chem. Res. 1989, 28, 1684-1688. [CrossRef]

35. Aussillous, P.; Quéré, D. Quick Deposition of a Fluid on the Wall of a Tube. Am. Inst. Phys. 2000, 12, $2367-2371$. [CrossRef]

36. Han, Y.; Shikazono, N. Thickness of liquid film formed in slug flow in micro tube. In Proceedings of the ECI International Conference on Heat Transfer and Fluid Flow in Microscale, Whistler, BC, Canada, 21-26 September 2008; pp. 21-26.

37. Liu, H.; Vandu, C.O.; Krishna, R. Hydrodynamics of Taylor Flow in Vertical Capillaries: Flow Regimes, Bubble Rise Velocity, Liquid Slug Length, and Pressure Drop. Ind. Eng. Chem. Res. 2005, 44, 4884-4897. [CrossRef] 
38. Abiev, R.S.; Lavretsov, I. Intensification of mass transfer from liquid to capillary wall by Taylor vortices in minichannels, bubble velocity and pressure drop. Chem. Eng. Sci. 2012, 74, 59-68. [CrossRef]

39. Rocha, L.; Miranda, J.; Campos, J. Wide range simulation study of taylor bubbles in circular milli and microchannels. Micromachines 2017, 8, 154. [CrossRef]

40. Hirt, C.W.; Nichols, B.D. Volume of fluid (VOF) method for the dynamics of free boundaries. J. Comput. Phys. 1981, 39, 201-225. [CrossRef]

41. Youngs, D.L. Time-dependent multi-material flow with large fluid distortion. Numer. Methods Fluid Dyn. 1982, 273-285.

42. Brackbill, J.U.; Kothe, D.B.; Zemach, C. A continuum method for modeling surface tension. J. Comput. Phys. 1992, 100, 335-354. [CrossRef]

(C) 2020 by the authors. Licensee MDPI, Basel, Switzerland. This article is an open access article distributed under the terms and conditions of the Creative Commons Attribution (CC BY) license (http://creativecommons.org/licenses/by/4.0/). 